\title{
Indocyanine green fluorescence angiography of the choroid
}

\author{
A. CRAANDIJK AND C. A. VAN BEEK \\ From the Eye Hospital, The Hague, The Netherlands
}

The introduction of fluorescence angiography by Novotny and Alvis (1960) was an important advance in our knowledge of the circulation and pathology of the retina. Our knowledge of the choroidal circulation is limited to cases of disease of the pigment epithelium and the choriocapillaris. Not only is visible light unable to penetrate the pigment epithelium and macular pigment but also fluorescein in the choriocapillaris rapidly leaks, obliterating vessel detail. The peculiar anastomosing of the vessels of the choroid parallel to the optic axis also contributes to the relative invisibility of the choroid.

Kogure, David, Yamanouchi, and Choromokos (1970) first demonstrated choroidal angiography in the Owl monkey using indocyanine green (ICG) with the 'false colour' infrared aero film. Drawbacks to this method are the high concentration of indocyanine green needed, the arterial injections, and the inconsistency of the false colour film. Hochheimer (1971) used black and white infrared film, and injected the indocyanine green intravenously in the cat. Flower (1972) showed that intravenous ICG absorption angiography could be performed repeatedly in man. Infrared light absorbed by ICG shows the filling of the large choroidal and retinal vessels but little filling of the arteries and capillaries.

Flower and Hochheimer (1973) described a method of ICG fluorescence angiography in which the arterial and capillary dye phases could be seen. A year later Flower (I 974) developed a camera which simultaneously records fluorescein, ICG absorption, and ICG fluorescence angiography. An extensive modification of the Zeiss fundus camera with two beam splitters, an intermediate relay lens, and three Nikon cameras was used in such a manner that the passage of the dye fronts could be recorded simultaneously on the three different films. From this it became apparent that the larger retinal and choroidal vessels are seen best on ICG absorption angiographies, whereas the smaller choroidal vessels are seen best on ICG fluorescence angiograms.

Address for reprints: A. Craandijk, MD, Eye Hospital, Leyweg 295, The Hague, The Netherlands
Our study was designed to reproduce Flower's results and to assess the potential clinical usefulness of ICG fluorescence angiography. Since we intended to record only fluorescence angiograms we were able to avoid the expense of extensive modifications of our camera.

\section{Method}

A Zeiss fundus camera was fitted with an exciter filter (interference filter with a peak at $777.5 \mathrm{~nm}$ ) in the diaphragm disc in front of the flash tube (Fig. I). The barrier filter (interference filter with a peak at $854^{\circ} 9$ $\mathrm{nm}$ ) fitted in a Zeiss $30^{\circ}$ conus in front of the film in such a manner that the camera body was attached over the conus. The film (Kodak high speed infrared sensitive black and white film, HIE-r 35-20) was developed in Kodak D-19 developer at $20^{\circ} \mathrm{C}$. The Beam flash unit was set at $600 \mathrm{~W} / \mathrm{s}$. Indocyanine green $25 \mathrm{mg}$ was diluted with aqueous solvent so that $2 \mathrm{ml}$ of ICG could be injected. The camera was firing at the rate of $\mathrm{I}$ frame/0.7 $\mathrm{s}$ and was started 8 seconds after the intravenous injection. The passage of the dye front could not be seen and there were only 20 frames available on the film.

\section{Results}

Several sets of ICG angiograms were compared with fluorescein angiograms which had been

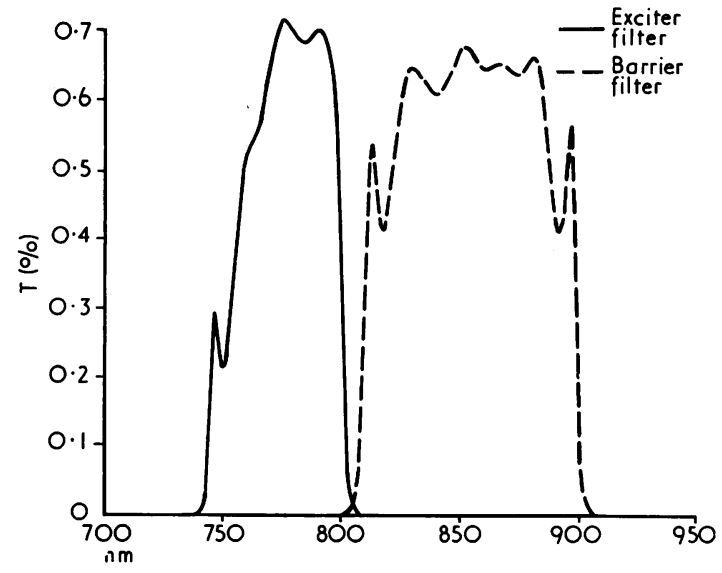

FIG. I Transmission curve of the ICG exciter and barrier filters 

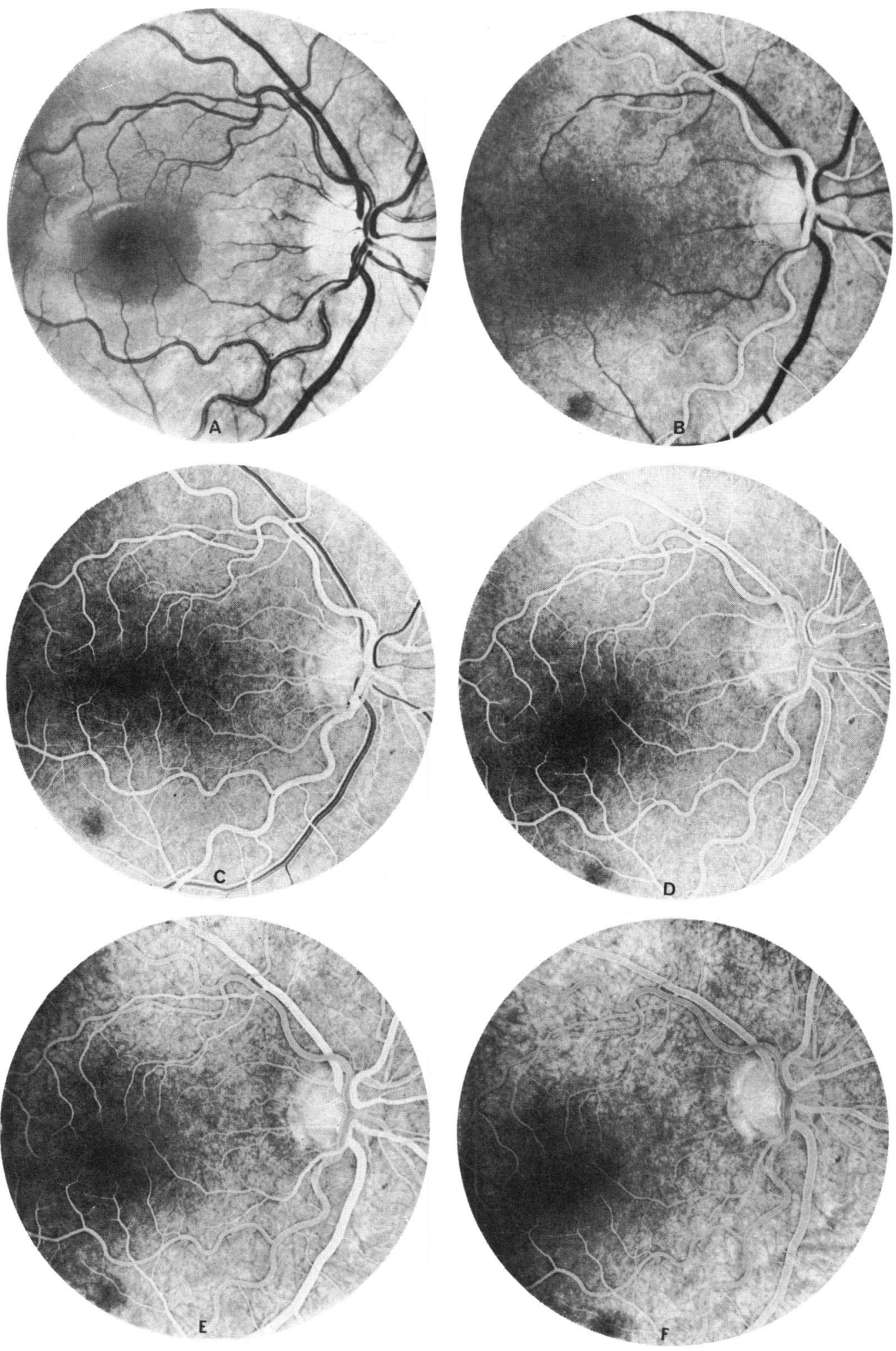

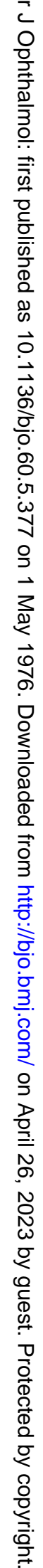



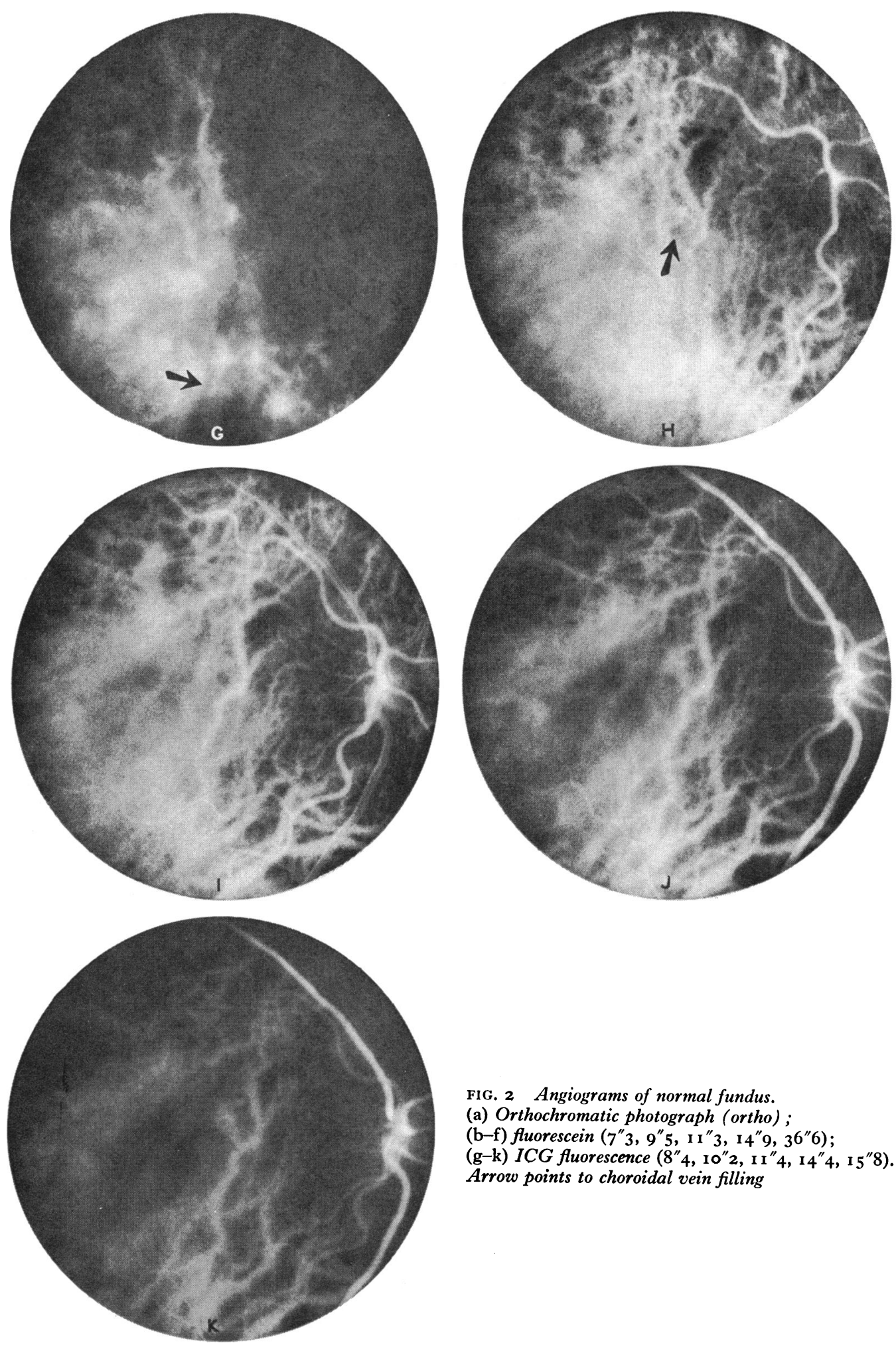

FIG. 2 Angiograms of normal fundus.

(a) Orthochromatic photograph (ortho);

(b-f) fluorescein (7"3, 9"5, I I"3, 14"9, 36"6);

(g-k) ICG fluorescence (8"4, $\left.\mathrm{IO}^{\prime \prime} 2, \mathrm{II}^{\prime \prime} 4, \mathrm{I}^{\prime \prime} 4, \mathrm{I} 5^{\prime \prime} 8\right)$.

Arrow points to choroidal vein filling 

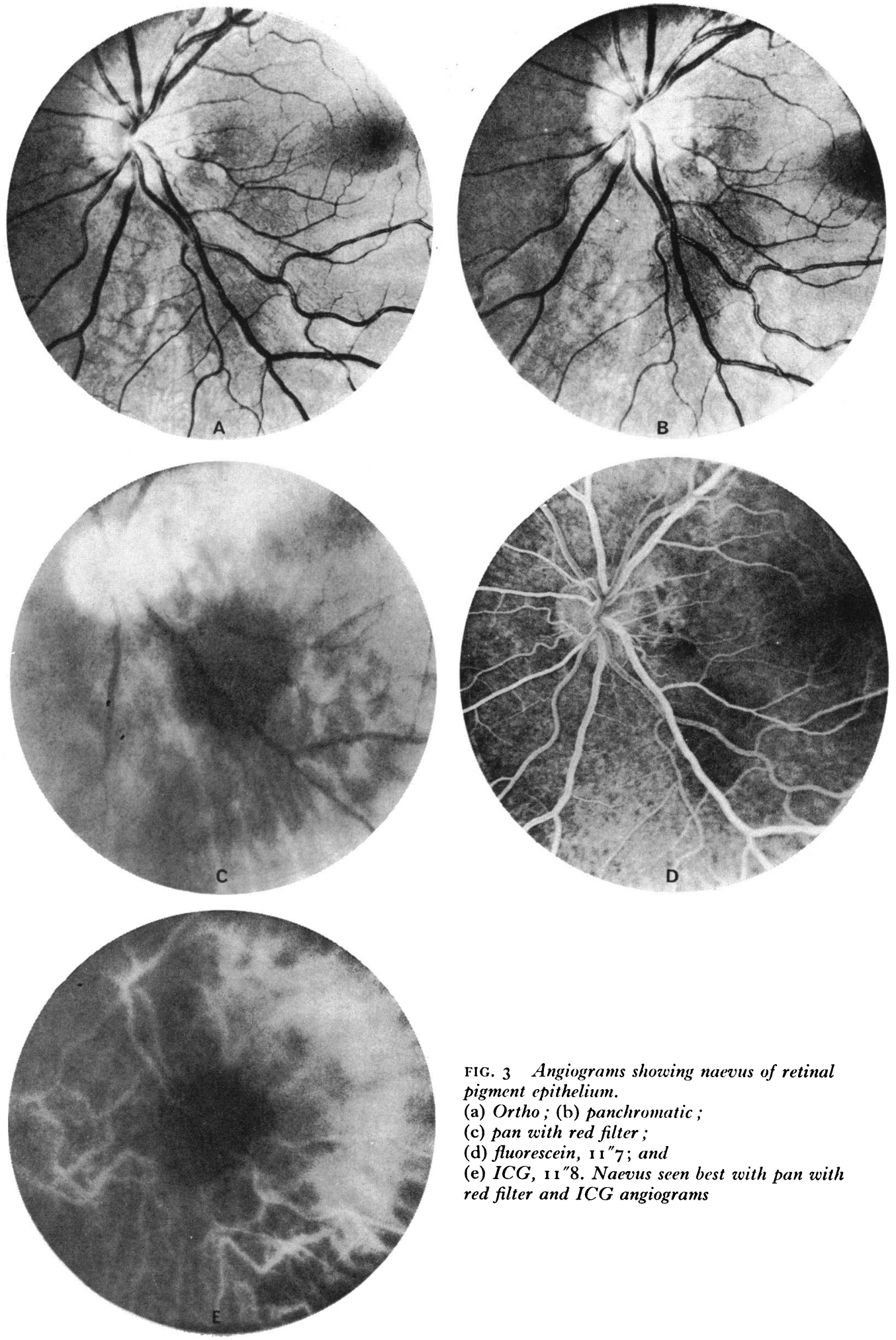

FIG. 3 Angiograms showing naevus of retinal pigment epithelium.

(a) Ortho; (b) panchromatic;

(c) pan with red filter;

(d) fluorescein, I I"7; and

(e) ICG, I I"8. Naevus seen best with pan with red filter and ICG angiograms 

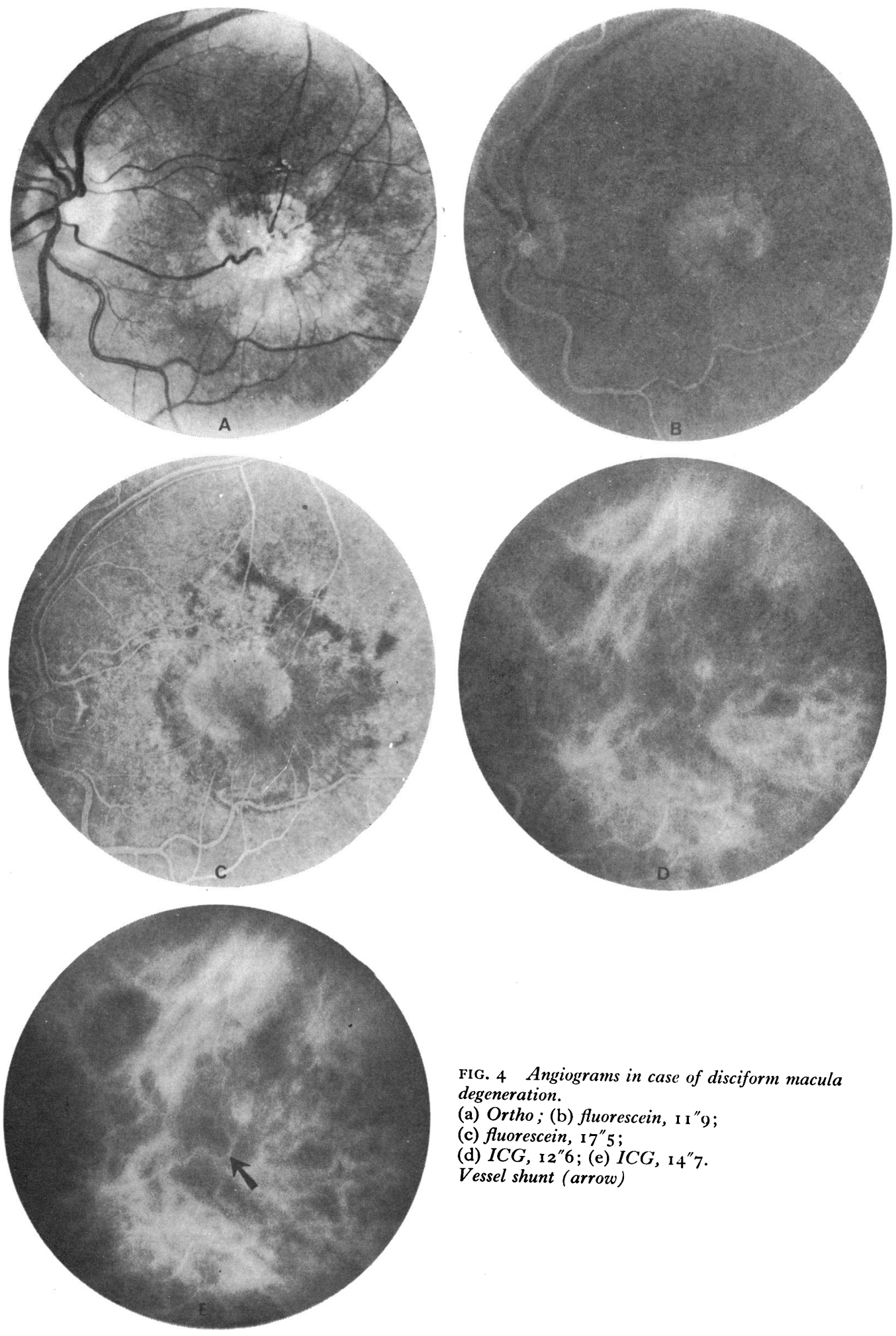

FIG. 4 Angiograms in case of disciform macula degeneration.

(a) Ortho; (b) fluorescein, I I"9;

(c) fluorescein, I 7 " 5 ;

(d) $I C G, 12$ "6; (e) $I C G, 14$ "7

Vessel shunt (arrow) 

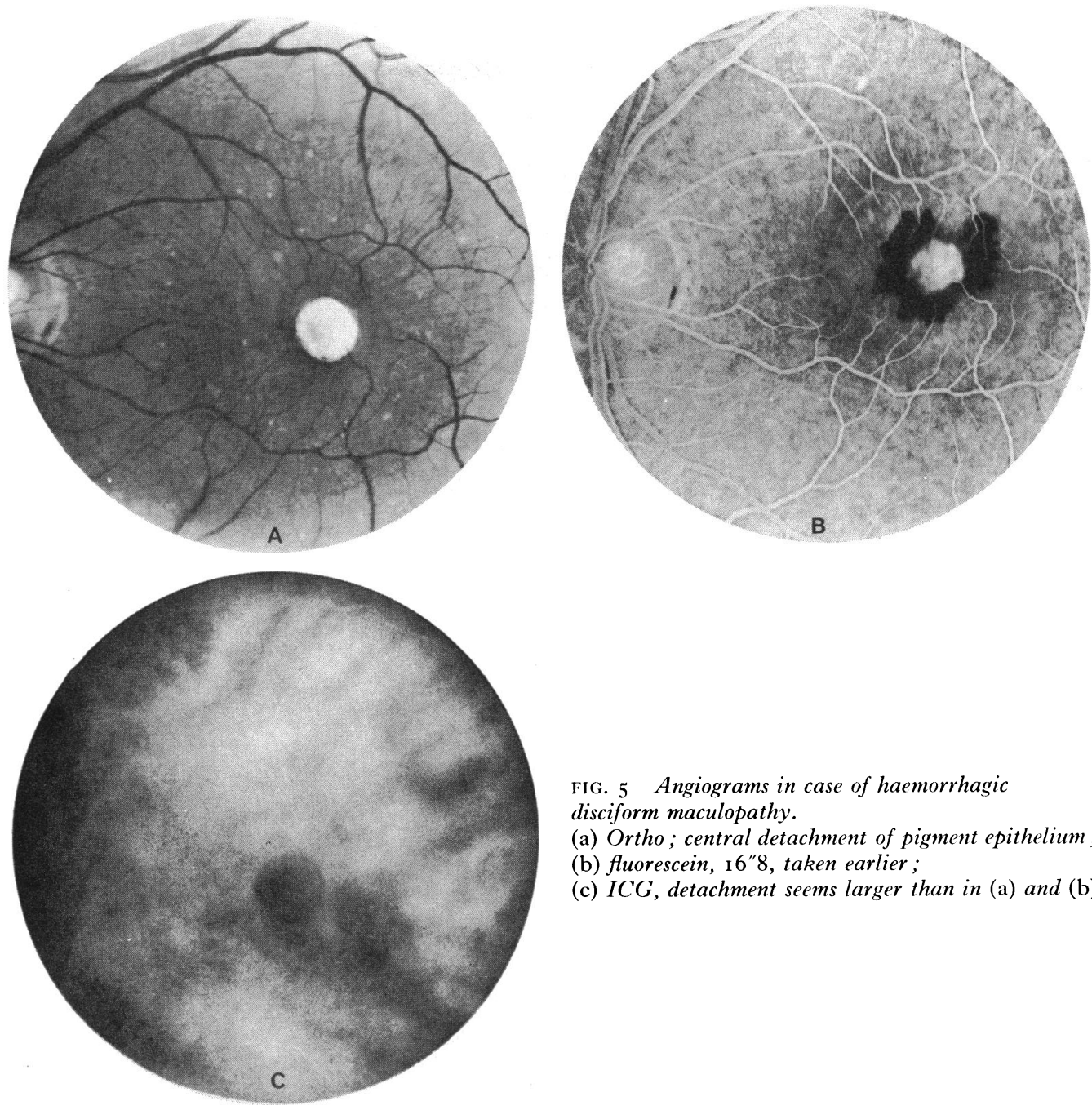

FIG. 5 Angiograms in case of haemorrhagic disciform maculopathy.

(a) Ortho ; central detachment of pigment epithelium ;

(b) fluorescein, I6"8, taken earlier ;

(c) ICG, detachment seems larger than in (a) and (b)

made immediately before. Our findings accorded with those of Orth, Patz, and Flower (1976). Since ICG angiograms are not easily interpreted we took an orthochromatic angiogram of a normal fundus in an I8-year-old girl together with fluorescein and ICG fluorescence angiograms of the same fundus for comparison (Fig. 2). The two main differences of ICG from fluorescein angiography are: first, ICG filling begins at the macula, and, secondly, the optic disc does not fluoresce. In the ICG angiogram at $8 " 4$ (Fig. $2 g$ ) the dark line (arrow) is a choroidal vein with maximum filling at $14^{\prime \prime} 4$ (Fig. 2j). The central dark spot persists throughout the series and corresponds with a pigmented spot in the fluorescein angiograms at I4"9 (Fig. 2e).

Fig. 3 shows a naevus of the pigment epithelium in a 20 -year-old man. On orthochromatic film the naevus is barely discernible (Fig. $3 a$ ), on panchromatic film it is detectable (Fig. $3 b$ ), but on the panchromatic film with red filter it is clearly delineated (Fig. $3 c$ ) (Craandijk and Aan de Kerk, N 1969). On the fluorescein angiogram the naevus is partly seen (Fig. $3 d$ ). The ICG angiogram clearly shows the naevus blocking the underlying choroidal fluorescence, whereas the retinal vessels are seen passing over the naevus (Fig. $3^{e}$ ).

Fig. 4 shows disciform macular degeneration in a 75-year-old man. Two retinal veins are seen to disappear in the centre of the disciform lesion. In the fluorescein angiograms these veins can no longer be traced to the centre owing to the fluorescence of the disciform lesion itself (Fig. $4 b, c$ ). Also note the pigmentations above the disciform lesion. In the ICG angiograms the retinal veins seem to form a shunt with a choroidal vessel 

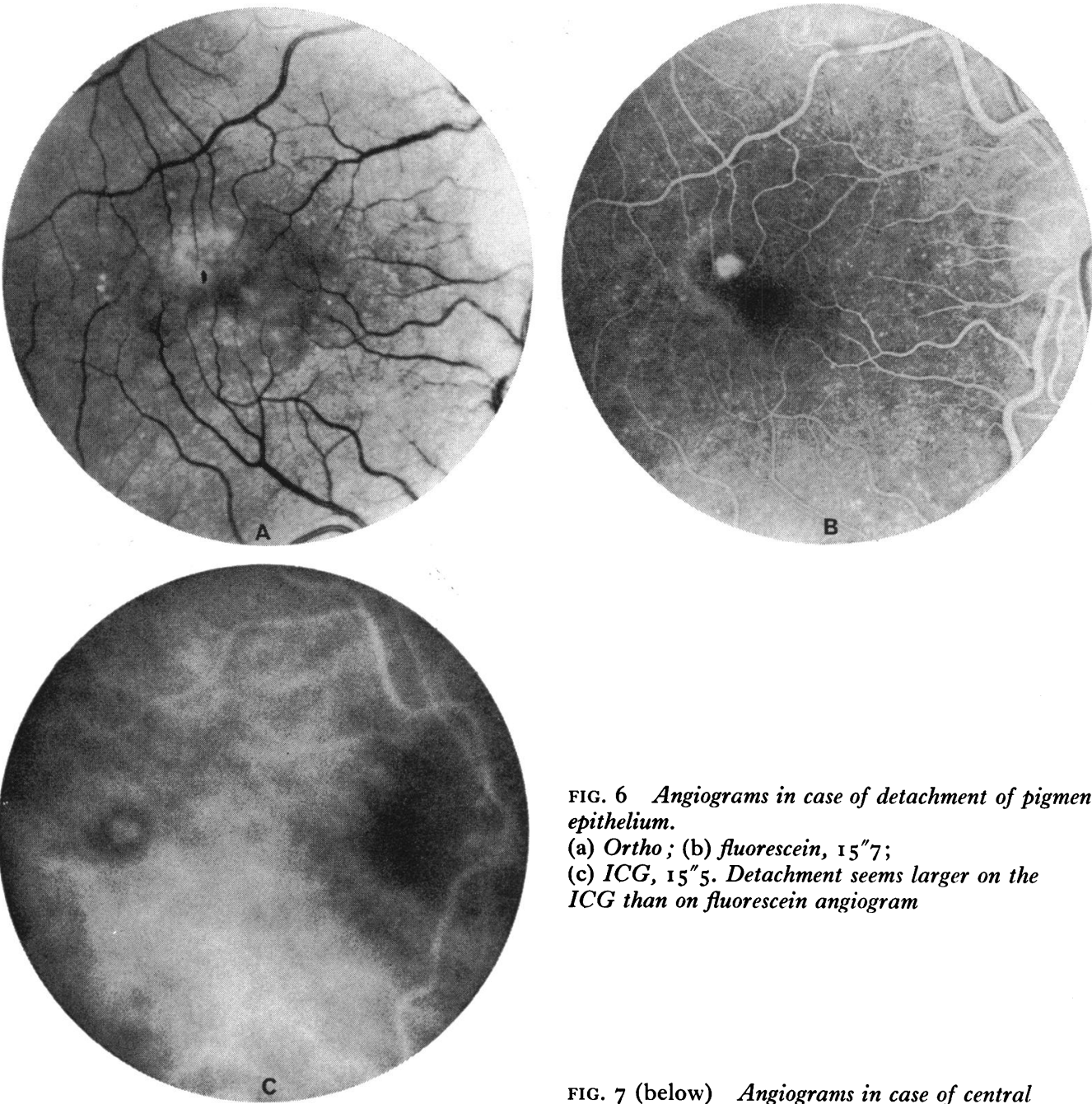

FIG. 6 Angiograms in case of detachment of pigment epithelium.
(a) Ortho; (b) fluorescein, I 5"7;
(c) ICG, 15" 5. Detachment seems larger on the
ICG than on fluorescein angiogram

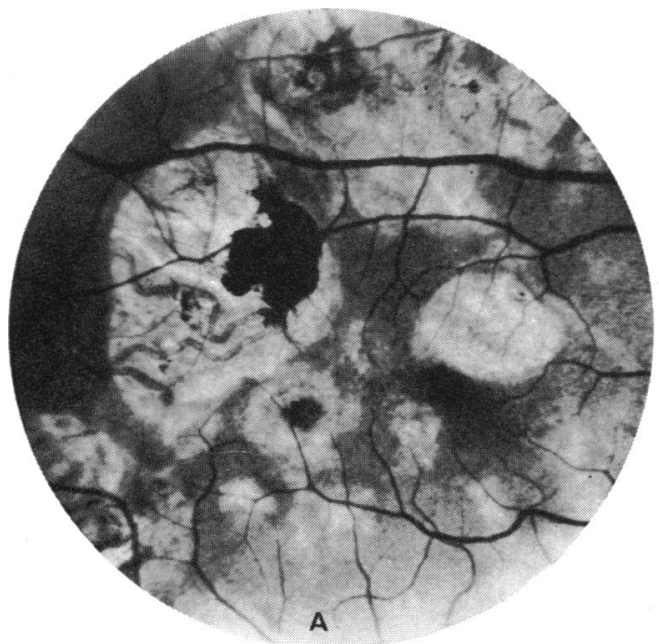

FIG. 7 (below) Angiograms in case of central chorioretinitis. (a) Ortho; (b) ICG, 12 "7.

Pigmentations block underlying choroidal fuorescence

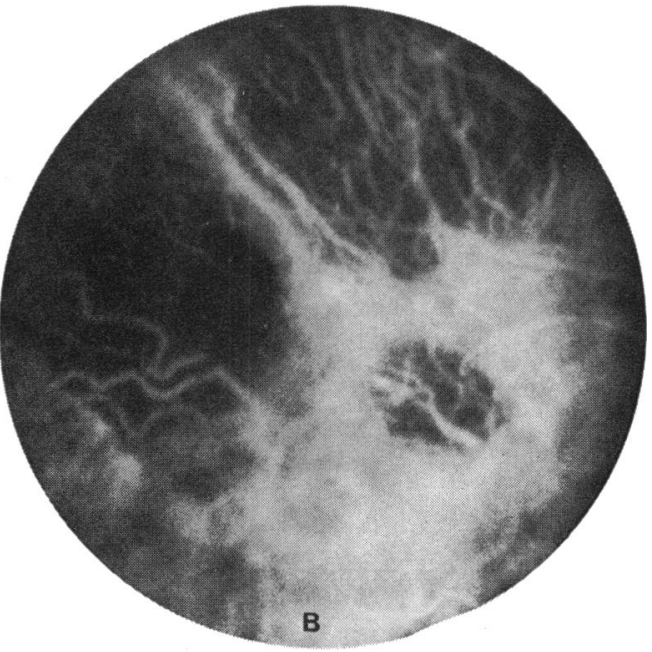


FIG. 8 Angiograms in case of Stargardt's disease.

(a) ortho; (b) fluorescein, 5"6;

(c) fluorescein, 13"6; (d) $I C G, 1_{1} 1$;

(e) ICG, 14 "6. Dark lesions on ICG angiograms do not correspond with pigmented lesions in bull's eye and may imply choroidal involvement 
(Fig. $4 d, e$ (arrow)). The pigmentations that were so clear in the fluorescein angiograms can be traced only with great difficulty in the ICG angiograms.

Fig. 5 shows a haemorrhagic disciform maculopathy in a 32-year-old man. The orthochromatic photograph shows the detachment of the pigment epithelium (Fig. 5a). Unfortunately the fluorescein angiogram taken a month earlier shows the detachment of the pigment epithelium with a collarette of haemorrhages (Fig. $5 b$ ). The ICG angiogram (Fig. 5c) shows the lesion to be larger than on the orthochromatic or on the earlier angiogram. These lesions correspond with those seen in cases of presumed ocular histoplasmosis.

Fig. 6 shows a detachment of the pigment epithelium in a 68-year-old man. The fluorescein angiogram clearly shows the detachment of the RPE (Fig. $6 b$ ), and on the ICG angiogram (Fig. $6 c$ ) this detachment seems to be larger.

Fig. 7 shows central chorioretinitis in a 60-yearold woman. The ortho angiogram shows a large pigmented lesion with choroidal vessels in the central atrophic area (Fig. $7 a$ ). On the ICG angiogram a much larger part than is to be expected is devoid of choriocapillaries (Fig. $7 b$ ). The pigmentations block the underlying fluorescence, whereas the larger choroidal vessels are seen in nearly all the atrophic areas.

Fig. 8 shows a case of Stargardt's disease in a 23-year-old man. The ortho angiogram shows the beaten bronze atrophy with the bull's eye in the centre (Fig. 8a). The fluorescein angiograms clearly show the bull's eye aspect (Fig. 8b). The ICG angiograms show pigment lesions in the centre (Fig. 8c). These cannot be proliferated pigment epithelium since they would have blocked the fluorescence in the fluorescein angiogram. Though rare, the choriocapillaris or the choroid may be involved (Deutman, 1971).

\section{Discussion}

The difference between the fluorescein and the ICG angiograms is obvious from these cases. Fluorescein angiography gives a better resolution and a better filling of the retinal vasculature. The choroidal vasculature is best demonstrated on ICG fluorescence angiograms, and is not limited to cases of diseased pigment epithelium and choriocapillaris. Orth, Patz, and Flower (1976) found differential leakage of fluoressein and ICG dyes in certain cases of neovascularization. We have limited ourselves primarily to macular lesions which could show differences in the fluorescein and ICG angiograms. Interpreting ICG angiograms takes far longer than fluorescein angiograms, partly because relatively little is known about the normal choroidal circulation.

ICG angiography does not require a very expensive adaptation of the camera, it can be performed in a few minutes, and can be followed by fluorescein angiography by changing the camera body and the syringe. We certainly agree with Flower that many more patients will need to be studied by different investigators before the clinical usefulness of ICG fluorescence angiography is proved.

\section{Summary}

Indocyanine green fluorescence (ICG) angiography of the choroid gives better visualization of the choroidal vessels than does fluorescein angiography. We found that the detachment of the pigment epithelium seems bigger on ICG than on fluorescein angiograms, and pigmented lesions are more clearly delineated.

The exciter and barrier interference filters were supplied by Ditric Optics, 247R Maple Street, Marlboro, Massachussetts or 752, USA.

\section{References}

CRAANDIJK, A., and AAN DE KERK, A. L. (1969) Brit. F. Ophthal., 53, 568

Deutman, A. F. (1971) 'The Hereditary Dystrophies of the Posterior Pole of the Eye', p. I33. van Gorcum, Assen Flower, R. W. (1972) Amer. F. Ophthal., 74, 600 (1974) Ophthal. Digest, 36, 18

-

HOCHHEIMER, B. F. (197I) Arch. Ophthal. (Chic.), 86, 564

KOGURE, K., DAVID, N. J., YAMANOUCHI, U., and CHOROMOKOS, E. (1970) Ibid., 83, 209

NOVOTNY, H. R., and ALVIS, D. L. (1960) Amer. Y. Ophthal., 50, 176

ORTH, D. H., PATZ, A., and Flower, R. W. (1976) Eye, Ear, Nose, Thr. Monthly, 55, 4 